\title{
Médiévales
}

Langues, Textes, Histoire

75 | automne 2018

Traductions du Moyen Âge

\section{Traduire Ovide au XIV siècle : les amours de Mars et de Vénus au livre IV des Métamorphoses et de l'Ovide moralisé}

Translating Ovid in the fourteenth century: The love of Mars and Venus in Book IV of the Metamorphoses and the Ovide moralisé

\section{Marylène Possamaï-Perez}

\section{(2) OpenEdition}

\section{Journals}

Édition électronique

URL : https://journals.openedition.org/medievales/9181

DOI : 10.4000/medievales.9181

ISSN : $1777-5892$

Éditeur

Presses universitaires de Vincennes

Édition imprimée

Date de publication : 15 octobre 2018

Pagination : 81-96

ISBN : 978-2-84292-861-2

ISSN : 0751-2708

\section{Référence électronique}

Marylène Possamaï-Perez, «Traduire Ovide au xıv siècle : les amours de Mars et de Vénus au livre IV des Métamorphoses et de l'Ovide moralisé », Médiévales [En ligne], 75 | automne 2018, mis en ligne le 15 octobre 2019, consulté le 22 avril 2022. URL : http://journals.openedition.org/medievales/9181 ; DOI : https://doi.org/10.4000/medievales.9181 
Marylène Possamaï-Perez

\section{Traduire Ovide au XIV siècle : les amours de Mars et de Vénus au livre IV des Métamorphoses et de l'Ovide moralisé}

Le récit des amours adultères de Mars et de Vénus est donné au livre IV des Métamorphoses et de sa «traduction » en langue romane, l'Ovide moralisé en vers du début du XIV ${ }^{\mathrm{e}}$ siècle. Mais alors que le poème latin livre un récit de vingt-six vers, son hypertexte roman déroule l'histoire sur cent quatre vers, ce qui constitue une amplification par rapport à la proportion habituelle de cette traduction : celle-ci utilise généralement un couplet d'octosyllabes à rimes plates pour rendre un hexamètre dactylique du poème ovidien (le récit français devrait occuper cinquante-deux vers, il est donc exactement deux fois plus long).

En outre, si nous prenons «traduction » dans les différents sens qu'analyse Gérard Genette ${ }^{1}$, le moraliste fait suivre la « transposition formelle », ici « linguistique », d'une transposition « thématique », ou plutôt axiologique, suite hiérarchisée d'interprétations qui s'étend sur deux cent soixante-huit vers et enchaîne trois types d'interprétation. Une première, de type «naturaliste » (vers 1488 à 1537), fait de Vénus et de Mars les figures des planètes qui portent leur nom, ainsi que les symboles des « humeurs », des tempéraments humains associés à ces planètes. Une deuxième interprétation « historique », au sens évhémériste, s'étend du vers 1548 au vers 1629 (le fabliau du triangle amoureux débouche sur une morale «sociale », qui conseille aux maris cocus de faire comme s'ils ne s'apercevaient pas de leur infortune, pour garder sur leur femme une autorité fondée sur la peur !). Enfin une interprétation qui est la seule à mériter sous la plume de l'auteur le nom d' «allégorie » occupe les vers 1630 à 1755 : c'est une longue leçon tropologique (celle du « quid agas », la façon dont l'homme doit se comporter, selon le dogme chrétien, pour gagner son salut).

1. G. Genette, Palimpsestes. La littérature au second degré, Paris, 1982, p. 291 sq. 
Les deux premières « expositions » sont des « relais sensibles », qui servent tout à la fois à «nettoyer » la fable de ses éléments inadmissibles pour la raison ou la morale, à lui donner un sens littéral acceptable, afin de pouvoir délivrer le sens spirituel (selon la théorie des « quatre sens de l'écriture ${ }^{2} »$ ), et à offrir au lecteur non averti une sorte de tremplin qui lui permette d'effectuer ce que Gilbert Dahan appelle «le saut herméneutique ${ }^{3}$ », des significations sensibles aux significations intelligibles.

La question que nous nous poserons à propos de cette version romane des Métamorphoses est celle de ses sources : de quels outils se sert l'auteur anonyme de l'Ovide moralisé pour traduire la légende des amours de Mars et de Vénus, pour l'amplifier conformément aux conseils des Arts poétiques des $\mathrm{XII}^{\mathrm{e}}$ et XIII ${ }^{\mathrm{e}}$ siècles (puisque l'amplificatio est le lieu essentiel de l'inventio de l'auteur médiéval) ? Quelles sont les sources utilisées en vue de donner différentes interprétations à la fable, conformément cette fois à la théorie de l'integumentum, qui pousse les auteurs médiévaux à rechercher la leçon morale sous le voile de la mythologie antique?

Trois niveaux sont à considérer pour notre étude, dont les deux premiers concernent plus spécialement la version romane de la légende, sa transposition linguistique, tandis que le troisième se rapporte à la transposition axiologique ou thématique : un niveau purement technique, celui du choix des mots de la traduction ; un niveau plus littéraire, celui du choix des éléments de l'amplificatio ; le niveau herméneutique enfin, qui concerne les interprétations de la légende.

\section{Le choix des mots}

\section{La traduction des Métamorphoses}

L'auteur a sous les yeux une version du texte d'Ovide, sans doute un manuscrit qui contient des gloses interlinéaires, comparable au manuscrit Città del Vaticano, BAV, Vat. lat. 1479 (f. 81r), que nous prendrons comme exemple puisqu'il date du XIII siècle. En effet, les gloses sont assez similaires d'un manuscrit à l'autre, comme on peut le constater en comparant par exemple le Vat. lat. 1479 avec les copies qu'étudie Frank T. Coulson, comme le Vat. lat. 1598 ou le manuscrit qu'il appelle $\mathrm{T}$ (soit le manuscrit 34 conservé au Centre de recherche Harry Ranson à Austin, Université du Texas) ${ }^{4}$.

2. H. DE LuBAC, Exégèse médiévale. Les quatre sens de l'Écriture, Paris, 1959-1964 (4 vol.) ; ID., "Sur un vieux distique. La doctrine du "quadruple sens" », dans Mélanges offerts au R. P. Ferdinand Cavallera, Toulouse, 1948, p. 347-366.

3. G. Dahan, L'Exégèse chrétienne de la Bible en Occident médiéval (XII ${ }^{e}$-XIV ${ }^{e}$ s.), Paris, 1999, p. $435 s q$.

4. Voir F. T. Coulson, The Vulgate commentary on Ovid's Metamorphoses, Book I, Kalamazoo, 2015. 
C'est ainsi que, là où l'édition du poème latin par Georges Lafaye ${ }^{5}$ donne pour la narratrice de la fable le nom de Leuconoé, le Vat. lat. 1479 copie Leucothoé comme l'Ovide moralisé $e^{6}$. Mais pour traduire « orsa est » par «s'est avancie » ${ }^{7}$, le translateur anonyme néglige la suggestion d'un glossateur comme celui du Vat. lat. 1479 (« incepit»), qu'il reprend cependant lorsqu'il ajoute «Si ra la soie commencie ${ }^{8} »$. L'action du soleil, «primus [...] hic [...] deus » qui « videt [...] omnia primus », est traduite littéralement par «Cil diex, qui voit premierement / Quanqu'il a au monde ainz que nulz ${ }^{9}$ » et « adulterium Veneris cum Marte putatur / [...] vidisse » n'a pas besoin de glose pour être rendu par « S'aperçut que Mars et Venus / Fesoient ensamble avoutire ${ }^{10}$.

Le translateur roman reproduit encore assez fidèlement les mots : « at illi / et mens et quod opus fabrilis dextra tenebat / excidit ${ }^{11}{ }$, lorsqu'il écrit : « trop ot grant ire / Et trop durement s'esbahi. / L'œuvre qu'il forgoit li cheï ${ }^{12} »$. Il est peut-être aidé par des gloses interlinéaires telles que celles du Vat. lat. 1479, qui indiquent «faber erat » au-dessus de « opus fabrilis » et « cecidit» au-dessus de « excidit».

Pour prendre un dernier exemple, les vers qui décrivent le piège fabriqué par Vulcain sont rendus dans une forme proche de l'original : « extemplo » est bien traduit par « sans plus attendre », de même que « graciles catenas » par « unes chaenes [...] si grellettes », « ex aere » par «d'airain », « retiaque et laqueos » par « Plaines de laces et de giez », et « non summo quae pendet aranea tigno » par «C'onques de si subtive ouvraigne / Ne furent li las de l'iraigne ${ }^{13}$. Le translateur anonyme ne suit pas, par exemple, les suggestions d'un glossateur tel que celui du Vat. lat. 1479, qui pour «tigno » signale « chevron gall », c'est-à-dire « chevron en gallo-roman ». Mais lorsqu'il traduit «tenuissima [...] stamina » par « Ne fil de soie si deugiez », il peut au contraire s'appuyer sur une glose telle que celle du mot «stamina », pour lequel le Vat. lat. 1479 donne « fila ${ }^{14}$. De même, sa façon de rendre les mots « quae lumina fallere possent » par

5. Ovide, Metamorphoseon libri 15 (Les Métamorphoses, éd. et trad. G. LafaYe, Paris, 1969, t. I, l. IV, désormais Mét., v. 168).

6. Ovide moralisé, poème du commencement du quatorzième siècle, publié d'après tous les manuscrits connus par C. DE BoER, Amsterdam, 1915-1938, vol. II, 1. IV, (désormais Om), v. 1270 .

7. Mét., v. $167 ;$ Om, v. 1270.

8. Om, v. 1271.

9. Mét., v. 171-172; Om, v. 1283-1284.

10. Mét., v. 171-172; Om, v. 1285-1286.

11. Mét., v. 174-176 : «La raison du mari et l'ouvrage que sa main façonnait lui échappèrent en même temps » (trad. G. LAFAYE, p. 102).

12. Om, v. 1295-1297.

13. Mét., v. 176-179; Om, v. 1303-1307.

14. Mét., v. 178-179; Om, v. 1308. 
« Nulz homs ne les peüst trouver / N'apercevoir d'œil qu'il eüst » ${ }^{15}$, peut être soufflée par des gloses comme celles du Vat. lat. 1479, qui expliquent «lumina » par « visum» ou « fallere » par « decipere».

\section{Les autres sources}

Ce manuscrit glosé ne suffit pas à l'érudit qui traduit les Métamorphoses : il connaît aussi les mythographes qui l'ont précédé, et sans doute les versions en langue romane de l'Ars amatoria d'Ovide. En outre, on sait que l'auteur connaissait aussi les romans du XII siècle et aimait rivaliser avec eux ${ }^{16}$. On en trouve des traces assez nettes dans sa translation de la fable de Mars et de Vénus.

Par exemple, lorsqu'au vers 1291, le translateur présente Mars comme «Le dieu de bataille», il est peut-être redevable au Roman d'Eneas qui, relatant la fabula $^{17}$ de Mars et de Vénus, présente l'amant de la déesse au vers $4356^{18}$ par les termes « Mars, qui ert deux de bataille ». Mais l'indication est sans doute bien connue au Moyen Âge. Plus probant est peut-être l'exemple suivant : la description de la chaîne forgée par Vulcain, « unes chaënes $[\ldots]$ si soutilles $[\ldots]$ » que la toile de l'araignée n'a pas « fil de soie si deugiez ${ }^{19}$, s'inspire peut-être aussi du Roman d'Eneas, qui utilise l'adjectif «soltil » au vers 4361, et « dolgié » au vers 4362 : «mot an furent dolgié li fil».

Pour traduire le vers 181 des Métamorphoses, « lecto circumdata collocat arte », par le vers 1316, « De teulz las a le lit porpris », le translateur a pu réunir les mots des vers 577-578 du livre II de l'Ars amatoria («Mulciber obscuros lectum circaque superque / Disponit laqueos ${ }^{20} »$ ), ceux des premier et deuxième Mythographes du Vatican ${ }^{21}$, ceux du Roman

15. Mét., v. 177 : «qui auraient pu tromper les lumières »; Om, v. 1313-1314.

16. Il cite Benoît de Sainte-Maure, et a dû lire aussi le Roman de Thèbes et le Roman d'Eneas : voir M. Possamaİ, « La légende thébaine dans l'Ovide moralisé : un exemple de contamination des sources », dans O. Collet, Y. Foenr-Janssens, S. Messerli éd., « Ce est li fruis selonc la letre ». Mélanges offerts à Charles Méla, Paris, 2002, p. 527-545. Sans doute possible, Chrétien de Troyes est l'un de ses modèles littéraires : voir EAD., «La réécriture de la complainte de Didon», dans C. Füg-PIERREviLle éd., Éditer, traduire ou adapter les textes médiévaux, Lyon, 2009, p. 239-248 ; EAD., L’Ovide moralisé. Essai d'interprétation, Paris, 2006, p. 263-297.

17. C'est le mot qu'utilise F. Mora-Lebrun dans $L$ 'Énéide médiévale et la naissance du roman, Paris, 1994, passim.

18. Énéas, roman du XII ${ }^{e}$ siècle (Anonyme), éd. J. J. Salverda De Grave, Paris, 1985, 2 vol.

19. Om, v. 1304, 1305, 1308.

20. Ovide, Ars amatoria (L'art d'aimer, éd. et trad. H. BorneCQue, rev. P. HeuZé, Paris, 2011), 1. II, v. 577-578 : « Mulciber dispose des lacs invisibles autour et au-dessus du lit » (notre traduction).

21. Scriptores rerum mythicarum latini tres Romae nuper reperti, éd. G. H. Bode, Hildesheim, 1968 (désormais Myth. Vat.). Myth. Vat., I, 43 : « lectulum cinxit»; Myth. Vat., II, 30 : « lectum catenis circumcinxit »; $121:$ « lectulum cinxit ». 
d'Eneas enfin : «Antor son lit l'aparoilla » (v. 4364). C'est le premier et le troisième Mythographes ${ }^{22}$ qui ont pu être sollicités pour fournir les mots des vers 1320-1321 : «Et il se sont entr'enbracié, / Tantost sont pris et enlacié ».

\section{La liberté du traducteur:}

la richesse des mots romans et l'annonce de la moralisation

Le passage offre un vocabulaire dont la richesse n'apparaît dans aucune des sources examinées, particulièrement dans les passages que le translateur ajoute à Ovide.

Par exemple la description de l'action du soleil, « dont tous biens habonde, / Qui ses rais espant par le monde, / Dont tout eschaufe et enlumine » (v. 1276-1278), développe les mots d'Ovide « siderea qui temperat omnia luce ${ }^{23} »$. Le choix des mots « rais », « eschaufe », « enlumine » n'est pas particulièrement original mais, joints à l'idée de l'abondance de tous biens, ils sont en lien avec l'allégorie que le moraliste donne toujours à Phébus, celle du Christ. Les participes qui, aux vers 12801281, amplifient le seul « cepit amor Solem » du vers 170 du poème latin, « souspris », « desjuglez ${ }^{24}$ », « avuglez », insistent sur la condamnation implicite de l'amour trompeur. Sans doute, une glose interlinéaire telle que celle du Vat. lat. 1479, qui interprète « cepit amor » par « id est decepit », a pu jouer un rôle, mais on sait que cette condamnation est l'un des thèmes favoris de notre moraliste dans ses interprétations ${ }^{25}$, et elle sera reprise dans la lecture tropologique de la fable de Mars et Vénus, aux vers 1640-1731.

La dénonciation de l'adultère s'enrichit dans la version romane des mots « $\operatorname{ahonter}^{26}$ » et « $\operatorname{acointier}^{27}$ » (v. 1289 et 1291), qui ne traduisent aucun mot latin. Quant à la réaction de Vulcain, elle est largement amplifiée dans la version romane, qui utilise les mots « duel », « corage », « enrage » (v. 1298-1299), sans équivalents dans le poème d'Ovide, ni dans aucune des sources, latines ou vernaculaires, auxquelles le translateur a pu puiser

22. Myth. Vat., I, 43 : «implicati sunt »; Myth. Vat., III, 11, 5, Venus : « eos coëuntes ligans ».

23. Mét., v. 169: «dont l'astre lumineux règle la vie de l'univers » (trad. G. LAFAYE, p. 101).

24. «Desjugler» signifie «Priver de qqch par tromperie» (étymon : «joculari »). Le mot est employé dès le XII siècle, mais il n'est pas d'un emploi extrêmement fréquent. Le dictionnaire de Tobler-Lommatzsch $(T L)$ et celui de Godefroy $(G d f)$ citent tous deux un exemple tiré du ms. de l'Arsenal de l'Ovide moralisé (ms. Paris, Bibliothèque de l'Arsenal, 5069).

25. Voir par exemple la digression sur l'amour dans la version de la fable de Philoména, au livre VI, v. 393 à 448.

26. Si le $T L$ en trouve un exemple dans le Tristan de Béroul, au v. 268 de l'édition d'E. Muret (Paris, 1903), le Dictionnaire électronique de Chrétien de Troyes (DÉCT) n'en donne aucun exemple, et il semble que le mot ne soit guère employé avant le XIII siècle.

27. "Acointier » est en revanche d'un emploi fréquent dès le début du XII siècle. C'est l'un des mots du vocabulaire courtois. 
par ailleurs. De même, les termes de «malice » ou de « forfait » (v. 13011302) ajoutent à l'hypotexte ovidien un jugement de valeur sur le piège ourdi par Vulcain : ce jugement moral rappelle que, dans l'Ovide moralisé, Vulcain est souvent interprété comme le diable ${ }^{28}$. Le fonctionnement du piège est explicité aux vers 1322-1325, en particulier au moyen d'un jeu sur « astraignant - destraignant », et les plaisanteries des dieux invités à contempler l'acte d'adultère sont définies non seulement par le mot « fable », mais aussi par « bourde » et «murmurement » (v. 1336-1337) : ces termes n'ont de correspondant dans aucune des sources examinées, et semblent d'ailleurs assez tardifs ${ }^{29}$. Cet ensemble de mots permet au translateur une description plus approfondie des ressorts psychologiques, qu'il imite des romanciers courtois du XII ${ }^{\mathrm{e}}$ siècle, mais qui préparent aussi ses interprétations morales.

\section{Les éléments de l'amplification}

Les quelque vingt-cinq hexamètres des Métamorphoses ${ }^{30}$ sont rendus par plus d'une centaine d'octosyllabes dans la version romane ${ }^{31}$. Si, comme nous le disions, l'on tient compte du fait qu'il faut le plus souvent un couplet d'octosyllabes pour traduire un hexamètre, le récit de la fable est deux fois plus long en français qu'en latin. Quelles sont les sources de cette amplification?

\section{Un récit des Métamorphoses amplifié grâce à l'Ars amatoria}

L'auteur du XIV ${ }^{\mathrm{e}}$ siècle connaissait les autres œuvres d'Ovide répandues au Moyen Âge et les a utilisées pour amplifier et éclairer les récits souvent très condensés des Métamorphoses ${ }^{32}$. Pour la fable de Mars et de Vénus, c'est sans doute l'Ars amatoria qui a aidé le translateur à développer en particulier les détails « coquins » ou misogynes.

On remarque ainsi une amplification du vers « illi iacuere ligati / turpiter » dans la traduction par «Quant cil se virent nuz et pris, / Si sont honteux et entrepris, / Qui dou forfet sont pris corpable » : l'ajout de la mention de la nudité peut s'expliquer par les mots de l'Ars amatoria (1. II,

28. Voir par exemple $\mathrm{Om}$, II, 2888-2891.

29. Les exemples donnés dans le $T L$, le $G d f$ ou le Dictionnaire du moyen français électronique (ATILF 2015) datent des XIII ${ }^{\mathrm{e}}-\mathrm{XV}^{\mathrm{e}}$ siècles, ces mots sont rares chez Chrétien de Troyes par exemple.

30. V. 167-192.

31. V. 1268-1371.

32. Il utilise en particulier les Héroïdes : on en a un exemple au livre IX, où l'Hérö̈de IX de Déjanire à Hercule explique la confusion entre Yolé et Omphale. 
v. 580 : «Impliciti laqueis nudus uterque iacent ») ${ }^{33}$. De la même façon, les vers 1338-1341, qui développent à l'envi l'épisode du « rire inextinguible des dieux », pourraient être débiteurs des vers 585-586 du livre II de l'Ars amatoria : "Hic aliquis ridens "in me, fortissime Mavors, / Si tibi sunt oneri, vincula transfer !" ait ${ }^{34} »$; et l'insistance sur la honte d'être vus nus et en position fâcheuse dans les vers « Par tot fu la chose seüe / Que Vulcanz avoit prise nue / Sa feme aveucques Mars gesant »(1342-1344), a pu être inspirée par la description complaisante de l'Ars : «Non vultus texisse suos, non denique possunt / Partibus obscenis opposuisse manus ${ }^{35} »$. Un autre vers ajouté aux Métamorphoses, le vers 1346 («Trop en fu Venus adolee») vient peut-être du vers 582 du livre II de l'Ars : «Vix lacrimas Venerem continuisse putant ${ }^{36} \gg$.

C'est aussi l'Ars amatoria qui a pu fournir la matière de l'amplification des vers 1347-1354, qui développent les sentiments de Vénus après l'aventure, en des termes d'une misogynie bien médiévale, mais souvent légitimée au Moyen Âge par la conviction que l'Ars était un traité d'amour sérieux ${ }^{37}$ : une fois sa liaison adultère publiquement révélée, la déesse, qui est femme, perd toute honte et toute crainte de son mari. Ces vers, ainsi que la mention de la douleur de Vulcain au vers 1355 de l'Ovide moralisé (« Trop convint puis Vulcan doloir »), ont pu être inspirés par l'apostrophe que le poète latin adresse à Vulcain à la fin du récit de la fable dans l'Ars amatoria : «Hoc tibi pro facto, Vulcane : quod ante tegebant, / Liberius faciunt, ut pudor omnis abest ${ }^{38} »$ (1. II, v. 589-590). Remarquons cependant qu'Ovide associe les deux amants dans cette disparition de la honte et de la crainte : le poète médiéval en charge la seule déesse...

\section{Autres sources}

Parmi les romans du XII ${ }^{\mathrm{e}}$ siècle qui ont pu fournir au translateur des éléments d'amplification narrative, c'est le Roman d'Eneas qui, on l'a dit, donne une version de la fabula de Mars et Vénus dont on peut retrouver la trace dans le poème du XIV ${ }^{\mathrm{e}}$ siècle.

33. On pourrait penser aussi aux mots des Fabulae d'Hygin (au chapitre « Vulcanus » : « ille eos nudos cubantes vidit»), mais les Fabulae n'ont guère circulé au Moyen Âge : nous n'en avons pas conservé de manuscrit médiéval.

34. «Celui-ci dit en riant : "Très vaillant Mars, si ces chaînes te pèsent, donne-lesmoi !" » (notre traduction).

35. L. II, v. 582-583 : « Ils n'ont pas pu couvrir leurs visages, ni poser leurs mains sur leurs parties honteuses $»$ (notre traduction).

36. « On pense que Vénus contint difficilement ses larmes » (notre traduction).

37. Cf. M. Desmond, Ovid's Art and the Wife of Bath : the Ethics of Erotic Violence, Ithaca/New York/Londres, 2006.

38. «Voilà ton salaire pour ton action, Vulcain : ce qu'ils cachaient jusque-là, ils le font plus librement, une fois toute pudeur envolée !» (notre traduction). 
Ainsi le développement de la saynète dans laquelle les dieux se gaussent des amants - et du mari cocu à la fois, a pu aussi s'inspirer des vers 4372-4373 de l'Eneas : « por quant s'en i ot il de teus / qui volsissent estre alsement / laciez o li estroitement ». L'ajout de la dolor de Vénus au vers 1346 peut venir du vers 4375 de ce même roman : «La deesse molt s'en marri ». La haine de Vénus envers Vulcain après la honte publique qu'il lui a fait subir, absente de l'hypotexte ovidien, est décrite dans le Roman d'Eneas aux vers 4376-4378 : « et son seignor molt anhaï ; / onc puis ne li mostra amor / ne bel sanblant jusqu'a cel jor ». Les vers 1358-1360 de l'Ovide moralisé font écho à ces vers du Roman : "Quar la dame tant l'en haï / Qu'el le lessa tout estrahi, / Si relenqui sa compaignie».

On peut aussi citer le vers 1370, que le translateur reprend au Roman de la Rose : « se duelent le leal amant ». Et le couplet sur la douleur de Vulcain, aux vers 1298-1305, doit sans doute beaucoup à des vers de Chrétien de Troyes : des phrases telles que «Tel duel en ot en son corage / Que bien petitet qu'il n'enrage » (v. 1298-1299), ont pu être soufflées à l'auteur du $\mathrm{XIV}^{\mathrm{e}}$ siècle par des vers comme ceux du Chevalier de la Charrette : "Car tant a duel et ire et rage / Qu'a bien petit que il n'anrage » (v. 7069-7070) ${ }^{39}$. La célébrité de Chrétien de Troyes était telle que le public du XIV siècle devait repérer immédiatement ces échos.

\section{Les éléments propres à l'auteur}

L'auteur du XIv ${ }^{\mathrm{e}}$ siècle ajoute à son hypotexte, aux vers 1310-1311, un commentaire élogieux sur Vulcain : «Trop fu subtilz et de grant sens / Qui tele oeuvre sot controuver ». On peut considérer que cet éloge est destiné aussi à celui qui met par écrit l'histoire de Mars et de Vénus ${ }^{40}$ : le filet de Vulcain, c'est l'écriture de la fable qui les prend au piège, qui les immobilise, qui les «éternise »; c'est même l'écriture qui les lie plus étroitement, en pérennisant l'histoire : comme les mailles du filet de Vulcain, les mots de l'auteur sont composés si solidement que « nulz rompre ne les peüst » (le v. 1314 est ajouté lui aussi aux Métamorphoses). En outre, les liens que notre poète tisse entre la fable antique et son ouvrage sont de la même façon aussi « subtils » qu' « indestructibles », parce qu'ils permettent de mettre en lumière le mensonge de la fable pour faire éclater la vérité chrétienne.

Il semble aussi que, dans son désir plus ou moins conscient de rivaliser avec les auteurs de romans du $\mathrm{XII}^{\mathrm{e}}$ siècle, et en particulier Chrétien de Troyes, son grand modèle en la matière, le poète du XIv siècle

39. Les Romans de Chrétien de Troyes, III, Le Chevalier de la Charrette, éd. M. RoQuEs, Paris, 1990.

40. Au XIV ${ }^{\mathrm{e}}$ siècle, les auteurs ont une pleine conscience de leur " métier », et de tels éléments à valeur métatextuelle ne sont pas rares dans le texte ; nombreux sont les indices qui autorisent une telle mise en abyme : voir M. Possamaï-Pérez, L'Ovide moralisé. Essai d'interprétation..., p. 623-716. 
amplifie particulièrement les passages de description des sentiments des personnages : nous avons déjà noté, à propos du choix des mots de la traduction, les ajouts concernant le récit de l'inamoramento du Soleil, «Qui de l'amour d'une meschine, / Fu si souspris et desjuglez, / Que touz en estoit avuglez » (v. 1279-1281), ou celui de la réaction de Vulcain apprenant la trahison de Mars et Vénus, aux vers 1298-1302. On peut rappeler également le couplet, plus moralisant cette fois, et misogyne, sur la disparition de la pudeur féminine et de la peur du mari chez la femme dont la liaison adultère a été découverte (v. 1342-1362) : il s'agit de vingt-et-un vers dont la source ne se lit ni dans les Métamorphoses ni dans aucun des textes antérieurs qui traitent de la fable de Mars et Vénus.

Mais ce sont bien sûr les interprétations qui suivent la transcription de la fable qui constituent la plus longue et la plus évidente des réécritures. Peut-on repérer des sources pour cette deuxième transposition?

\section{Les sources des interprétations}

Le moraliste du XIV $v^{\mathrm{e}}$ siècle propose deux «expositions » et une « allégorie » pour la fable de Mars et Vénus. On sait en effet qu'il étage et hiérarchise les sens " concrets $^{41}$ » et les sens spirituels ${ }^{42}$. Ici il propose deux sens concrets successifs, un sens de type «physique ${ }^{43}$ » (vers 1489-1537), et un de type « historique ${ }^{44} »($ vers 1538-1629). Pour le sens « allégorique », il s'agit d'une lecture tropologique, celle du « quid agas », de la façon de se comporter conformément au dogme chrétien pour obtenir le salut de son âme (v. 1632-1720).

\section{Les sources possibles}

Notre interprète a pu lire les mythographes qui l'ont précédé, mais il les interprète assez librement pour la fable de Mars et Vénus.

L'exposition ne doit rien à Fulgence, qui se concentre sur l'allégorie du Soleil et des cinq filles du Soleil punies par Vénus. Il en est de même

41. Rappelons que ces sens « concrets » permettent à la fable de recevoir des sens allégoriques, d'accéder par étapes aux vérités intelligibles, auxquelles seules le moraliste donne le nom d' «allégories ». Pour les premières interprétations, il parle d' ' expositions » ou de « sentences » et utilise les verbes « espondre », comme ici au vers 1488, ou « entendre ».

42. Les quatre sens de l'Écriture peuvent être dans l'Ovide moralisé distingués ou indifférenciés. Ce qui est certain, c'est qu'entre les vérités sensibles et les vérités intelligibles, l'interprète de l'Ovide moralisé effectue ce que Gilbert Dahan appelle le « saut herméneutique ». Sur toutes ces questions, voir M. Possamaï-Pérez, L'Ovide moralisé. Essai d'interprétation..., p. 363-493.

43. Ce sens est celui que les stoïciens proposent dès l'Antiquité pour les fables mythologiques.

44. Il s'agit cette fois de la méthode évhémériste. 
pour les premier et deuxième Mythographes du Vatican, qui insistent particulièrement sur l'une de ces filles, Pasiphaé.

L'interprétation de type physique n'est pas celle de Jean de Garlande, qui fait de Vénus le printemps, de Vulcain l'été, et de Mars l'automne : «Ver Venus est, estas Vulcanus, captus adulter / Autumnus nobis dans aliena bona ${ }^{45}$ ». L'interprète de l'Ovide moralisé retrouve les lectures qui font des dieux les figures des planètes. Mars et Vénus sont deux planètes, qui influent sur les humains en déterminant leurs humeurs : Mars, « planete errables, Plains de colerique nature » (v. 1489-1490), est celle qui influe sur l'humeur des colériques : «Si predomine aus corageus, / Aus mellis et aus irascibles » (v. 1495-1496). Vénus, planète chaude et humide (v. 1498), est la planète des sanguins (v. 1500). Vulcain est «l'outrageuse ardure » qui naît de la « jointure » de ces deux « étoiles » (v. 1519-1522).

Chez les Latins, Sénèque est l'héritier des conceptions cosmologiques des stoïciens. C'est ainsi qu'au Livre VII de ses Questions naturelles, où il traite des comètes, il évoque les planètes Mars et Vénus ${ }^{46}$ :

Encore maintenant il arrive fréquemment qu'une étoile se place sous la trace d'une étoile plus haute : parfois Saturne est au-dessus de Jupiter et Mars regarde d'en haut en droite ligne Vénus ou Mercure ; cependant ce n'est pas à cause de cette rencontre entre eux, lorsque la course d'une étoile est au-dessous de la course de l'autre, que se produit une comète; du reste cela arrive tous les ans (tous les ans en effet certaines étoiles sont en même temps situées dans le même signe [du zodiaque]).

Hygin, dans les Astronomica ${ }^{47}$, fait état de cette conjoncture entre les planètes Mars et Vénus, et la justifie par la fable, mais en en transformant les données ${ }^{48}$ :

45. Giovanni di Garlandia, Integumenta Ovidii, poemetto inedito del secolo XIII, éd. F. Ghisalberti, Messine/Milan, 1933, 1. IV, v. 185-186 : «Vénus est le printemps, Vulcain l'été, l'adultère captif est l'Automne qui nous donne des biens étrangers » (notre traduction). Cependant, dans une note à ces vers des Integumenta, Ghisalberti copie une glose qui se trouve dans le ms. Ambrosianus (Milano, Biblioteca Ambrosiana, N 254) et pourrait expliquer cette interprétation de l'Ovide moralisé (Ghisalberti lui-même signale cette correspondance) : «Venus et Mars sunt duo planete habentes interjectos. Venus vero benivolentia sua temperat malivolentiam Martis, et propter hoc fingitur quod adultera est Venus cum Marte. Unde habentur isti duo versus : "Ver...bona" (Integ. IV, v. 185-186). Je remercie Irene Salvo García d'avoir attiré mon attention sur cette note, qui donne une version particulière des Integumenta.

46. SÉnèque, Naturales quaestiones (éd. P. Oltramare, Paris, 1929, vol. II, 1. VII, De cometis, 12, 3) : «Etiam nunc frequenter stella sub altioris stellae uestigium uenit: et Saturnus aliquando supra Iouem est et Mars Uenerem aut Mercurium recta linea despicit, nec tamen propter hunc illorum concursum, cum alter alterum subit, cometes fit ; alioquin annis omnibus fieret (omnibus enim aliquae stellae in eodem signo simul sunt) (notre traduction).

47. Contrairement aux Fabulae, ce texte, qui contient nombre d'éléments mythologiques, a circulé au Moyen Âge.

48. Hygin, Astronomica, II, 42, 3 (éd. A. Le Boeuffle, Paris, 1983) : « Tertia est stella Martis, quam alii Herculis dixerunt, Veneris sequens stellam hac, ut Eratosthenes ait, de causa. Quod Vulcanus cum uxorem Venerem duxisset, et propter eius observantiam Marti 
La troisième étoile est celle de Mars, selon d'autres celle d'Hercule. Elle suit l'astre de Vénus, comme le dit Ératosthène, pour le motif suivant : Vulcain avait épousé Vénus et sa vigilance empêchait Mars d'arriver jusqu'à elle, au point qu'il semblait ne rien obtenir d'autre que la permission de marcher à ses côtés, comme son étoile. C'est pourquoi, comme il brûlait d'une passion véhémente, en guise d'explication au phénomène, on l'appela l'Astre du Feu.

Il faut donc imaginer que le moraliste du XIV ${ }^{\mathrm{e}}$ siècle a pu ajouter aux gloses de son manuscrit des Métamorphoses des éléments qu'il avait lus directement dans les Questions naturelles de Sénèque, ou indirectement à travers leur reprise par Hygin dans les Astronomica, et développer ces données pour son interprétation de type naturaliste de la fable de Mars, Vénus et Vulcain, puisque celle-ci se déploie sur cinquante vers (vers 1489-1537).

Pour ce qui concerne l'interprétation de type « historique », au sens où l'entend l'interprète de l'Ovide moralisé, celui d'évhémériste, elle a pu naître dans l'esprit de l'interprète à la lecture de l'Ars amatoria d'Ovide, mais aussi à celle du Roman de la Rose de Jean de Meun. Comme cela arrive souvent, cette « histoire » se présente comme un exemplum, c'est-àdire un récit exemplaire, qui en l'occurrence a plutôt des allures de fabliau : «Venus [...] Fu une dame bele et gente [...]» (vers 1538-1540). Ce goût pour les récits romanesques est tout à fait original, et le moraliste a beau affirmer qu'il ne cherche pas à faire de beaux vers ${ }^{49}$, il n'en manifeste pas moins des « tentations littéraires ${ }^{50}$ », et le vocabulaire employé rappelle les romanciers du XII ${ }^{\mathrm{e}}$ siècle : «[...] pour ce qu'elle outreement / Sormontoit en joliveté, / En amours et en gaieté, / Et en beauté toute autre fame [...]» (vers 1543-1545). Le triangle amoureux est l'une des thématiques favorites du fabliau, et il est établi sans ambiguïté :

Un mari, ce m'est vis,

Avoit vilain despit et vis,

Qu'el ne prisoit une cenele.

Entre mil amis ot la bele

Un vaillant et chevalereuz,

Sor tous fier et bateillereux [...]. (v. 1548-1552)

Les sources de l'interprétation « historique » sont particulièrement repérables pour le long passage de condamnation du mari jaloux (v. 15691629). Il y a sans doute là l'écho de la condamnation du jaloux par Ovide dans l'Ars amatoria dont nous avons cité les vers 589-590. Et cette

copia non fieret, ut nihil aliud adsequi videretur, nisi sua stella Veneris sidus persequi a Veneri impetravit. Itaque cum vehementer amore eum incenderet, significans e facto stellam Pyroenta appellavit » (notre traduction).

49. Voir les vers 4192 à 4212 du livre XV.

50. Sur cette question, voir M. Possamaï-Pérez, L’Ovide moralisé. Essai d'interprétation..., p. 239-251. 
condamnation de la jalousie se trouve aussi longuement développée dans le Roman de la Rose de Jean de Meun ${ }^{51}$ : le mari jaloux est «li fous » qui épie les amants (v. 13816), et Vulcain « mout fu fos quant fere l'osa, / car cil a mout po de savoir / qui seus cuide sa fame avoir » (v. 13820-13822) ; «Trop est fos maus que jalousie, / qui les jalous art et soussie» (v. 14168-14169). De même, les conseils surprenants que le moraliste du XIV siècle donne aux maris cocus - qui ont tout à gagner de feindre l'ignorance (« Face samblant qu'il ne le voie / Ne qu'il ne l'ait aperceü », v. 1603-1604) - sont très proches de ceux que Jean de Meun donne à Vulcain de façon rétrospective : « mes feinsist que riens n'en seüst, / s'il vousist avoir bele chiere / de Venus, que tant avoit chiere » (v. 14155-14157).

Cependant, malgré l'ampleur inégalée que l'interprète de l'Ovide moralisé donne à cette « istorial matire » et aux conseils de morale sociale pragmatiques et opportunistes pour les maris cocus, c'est lorsqu'on passe à « l'alegorie / que ceste fable signifie » (v. 1630-1631) que l'originalité de l'auteur de l'Ovide moralisé déploie sa pleine mesure.

\section{L'originalité du moraliste : la passion du prédicateur}

La condamnation de l'amour humain peut venir de moralistes précédents : par exemple, le troisième Mythographe du Vatican fait de Mars amoureux de Vénus l'image de la vertu corrompue par le plaisir amoureux ${ }^{52}$ :

Mars igitur complexu Veneris pollutus, id est, virtus libidinis illecibris corrupta, sole teste apparet, id est, tandem veritatis indicio rea esse cognoscitur. Quae quidem virtus prava consuetudine illecta vinclis constrictioribus ostenditur catenata.

Mars séduit par les baisers de Vénus, c'est la vertu corrompue par les charmes du désir. Le fait est révélé par le témoignage du soleil, c'est-à-dire que finalement la chose est connue par la preuve de la vérité. Enfin cette vertu séduite par une habitude de débauche est montrée enchaînée par des liens très serrés.

On retrouve cette idée exprimée de la même façon presque mot pour mot chez Arnoul d'Orléans ${ }^{53}$ :

51. Guillaume de Lorris et Jean de Meun, Le Roman de la Rose (éd. F. Lecoy, Paris, 1965-1970 [réimpr. 1982-1983]), v. 13805-13844, v. 14128-14171, v. 18031-18099.

52. Myth. Vat., III, 11, 5 (notre traduction).

53. F. Ghisalberti, Arnolfo d'Orleans : un cultore di Ovidio nel secolo XII, Milan, 1932, p. 157-234 : « On dit que Mars aima Vénus, parce qu'il arrive qu'un homme fort soit dissous en Vénus, c'est-à-dire que la corruption qui souille parfois la vertu par l'embrassement de Vénus, c'est-à-dire du plaisir, apparaît avec le témoignage du Soleil c'est-à-dire que la culpabilité est reconnue par le jugement de la Vérité. Cette vertu est entravée comme avec une chaîne par l'habitude dépravée d'une passion illicite » (notre traduction). 
Mars in adulterium

Mars Venerem dicitur amasse, quia aliquando vir fortis in Venerem dissolvitur, id est virtus aliquando corrupta amplexu Veneris id est libidinis Sole teste apparet id est in veritatis iudicio rea esse cognoscitur. Quae quidem virtus prava consuetudine illiciti fervoris quasi cathena constringitur.

Ce passage des Allegoriae d'Arnoul d'Orléans est recopié dans la marge du Vat. lat. $1479^{54}$. Ce sont de telles sources qui peuvent expliquer les vers 1640-1644:

Grief mort et grief destruction

Vienent par fornication.

Par luxure et par avoultire

Sont li pluisieur mis a martire

Et desnué de bones mours.

Mais cette reprise est rapide, et l'allégorie se développe sur plus de cent vers (1630-1732) : la passion du moraliste l'entraîne dans un long couplet contre l'amour charnel, qui utilise tous les procédés rhétoriques de la « pastorale », en particulier l'anaphore (celle du mot « amour », présenté comme abstractum agens). Le moraliste introduit cette diatribe par une définition en deux temps, un premier temps négatif : « je di qu'amours n'est ce mie » (v. 1646), et un second temps affirmatif, par le procédé de la correctio : «Ains est pechiez et vilonie » (v. 1647). La série des maux entraînés par «amour » ne compte pas moins de sept substantifs dont l'énumération est renforcée par la reprise de la copule et : «D'amours veint malz, paine et poverte / Ire, angoisse et mors et grief perte » (v. 16501651). L'amour terrestre fait partie de ces «métamorphoses négatives » qui entraînent l'homme vers le péché (ici la luxure) et la damnation. Le vocabulaire et les métaphores soulignent cette vision de l'amour. L'amour « fet faire » (v. 1672 et 1674), « Le cors gaste et gaste l'avoir ${ }^{55} »(1690)$; l'amour est comme un feu « qui art sans estaindre » (v. 1654), comme la soif de Narcisse, « Soif qui ja n'ert rassasiee » (v. 1668), comme une maladie, une «Fievre qui ja n'ert apaiee » (v. 1669), comme la mort (v. 1686) ou la Damnation, «Destructions d'ame et de cors » (v. 1659) ; l'amour est toujours en mouvement, « amours ne set estre en un point » (1676) ;

54. Città del Vaticano, BAV, Vat. lat. 1479, f. 81r, marge de droite : « Moralitas talis est : Mars Venerem amasse dicitur. Quia aliquando vir fortis in Venerem dissolvitur, ubi virtus aliquando complexa corrupta est amplexu* Veneris, id est libidinis, sole teste apparet, id est veritatis indicio rea esse cognoscitur. Que, siquidem virtus parva consuetudine illicito fervore, quasi cathena astringitur quam Vulcanus dicitur fabricavisse, quia maritus Veneris dicitur Vulcanus, quia Vulcanus interpretatur calor, et in calore corporis et ciborum Venus maxime nutritur » : transcription par Lisa Ciccone, que je remercie de m'avoir communiqué son travail. Cette interprétation sera reprise aussi par Giovanni del Virgilio : voir F. GHISALBERTI, Giovanni del Virgilio espositore delle Metamorfosi, Florence, 1931, p. 3-39.

55. Notons le chiasme autour de la reprise du verbe « gaste ». 
l'amour transforme l'aspect extérieur des amants, et de façon continuelle : « Or art, or cuit, or leche, or point » (1677), « Or a li amans froit, or chault, / Or est tristes et est hetiez, / Or est sains, or est dehetiez » (1681-1683); l'amoureux est comme sur la Roue de Fortune, «Or cuide avoir tout ce qu'il velt / Or en est plus loin qu'il ne selt » (1684-1685). «Les haulz abesse et les bas lieve, / Contre raison et contre droit» (1719-1720). L'amour est une folie, un piège, un serpent, bref, l'amour est diabolique - et l'on comprend la cohérence de cette condamnation de l'amour avec la fable qui décrit le piège de Vulcain : " Amour est forsen sans mesure, / Nasse et las et rois de fallace [...] / Vuivre que nulz ne puet destruire » (v. 1663-1666). «Amours prent et glue et enlace » (1716). L'amour est péché (1647), « Amours toute vertu efface » (1717). La diatribe se poursuit sur quatre-vingt-six vers (1646 à 1732), et la fréquente anaphore du mot « amour » en fait un écho inversé de l'hymne à la charité, dans la première épître aux Corinthiens de saint Paul (13, 4-7).

Cet enthousiasme du moraliste n'a pas d'équivalent chez les mythographes précédents, qui n'ont pas la visée homilétique de l'auteur du $\mathrm{XIV}^{\mathrm{e}}$ siècle ${ }^{56}$. La passion qu'il déploie dans ces couplets moraux ${ }^{57}$ l'entraîne plus loin aussi que les romanciers qui l'ont devancé et qu'il se plaît à imiter.

Ainsi, pour l'auteur anonyme de l'Ovide moralisé, traduire Ovide au début du XIV siècle exige d'être un érudit pour pouvoir « accomplir la matière ${ }^{58}$ », c'est-à-dire la compléter, l'éclairer, la vivifier aussi en lui donnant toute sa signification, en soulevant le voile de la fable pour révéler la vérité cachée. Cela demande de lire parfaitement le latin des Métamorphoses et des gloses interlinéaires et marginales qui sont copiées dans les manuscrits du poème à partir du XIII ${ }^{\mathrm{e}}$ siècle, de disposer des autres textes du poète augustéen, comme les Hérö̈des ou l'Ars amatoria, de connaître peut-être d'autres textes antiques comme ceux de Sénèque, mais aussi de recueillir

56. $C f$. M.-R. Jung, « Aspects de l'Ovide moralisé », dans M. Picone et B. Zimmermann éd., Ovidius redivivus. Von Ovid zu Dante, Stuttgart, 1994, p. 149-172 ; J.-Y. Tilliette, "L'écriture et sa métaphore. Remarques sur l'Ovide moralisé », dans L. Rossi éd., Ensi firent li ancessor. Mélanges de philologie médiévale offerts à Marc-René Jung, vol. II, Turin, 1996, p. 543-558 ; M. Possamaï-Pérez, L'Ovide moralisé. Essai d'interprétation..., p. 723 : « la tonalité voulue par l'exégète imite celle d'un prêche, à tout le moins celle d'un discours édifiant. Le ton est celui d'un éducateur, voire celui d'un prédicateur ».

57. Nous avons même pu parler de « souffle épique » à propos de ces diatribes fiévreuses : voir M. Possamaï-Pérez, "Le souffle épique dans l'Ovide moralisé », dans S. BazinTachella, D. de Carné, M. Ott éd., Le Souffle épique. L'esprit de la chanson de geste, Dijon, 2011, p. 435-444.

58. C'est l'expression qu'il utilise lorsqu'il introduit une amplification de la fable des Métamorphoses : $c f$. M. Possamaï-Pérez, « L'Ovide moralisé, ou la « bonne glose des Métamorphoses d'Ovide », dans Regards croisés sur la glose. Cahiers d'études hispaniques médiévales, 38 (2008), p. 181-206. 
les versions des romans antérieurs qui ont traité de la fabula. Cela nécessite enfin de manier les recueils mythographiques et les commentaires aux Métamorphoses des moralistes qui se sont succédé depuis l'aetas ovidiana du XII ${ }^{\text {e }}$ siècle $^{59}$. Mais ce n'est pas encore suffisant : pour redonner vie aux Métamorphoses, il faut leur insuffler un souffle nouveau. Ce souffle, c'est la foi chrétienne, c'est cette vérité suprême qu'il faut révéler sous le voile de la fable. Et la traduction, comprise dans son sens double, est tout entière destinée à ce but ultime : la première transposition, du latin au français, va souligner les mots et les motifs en lien avec la deuxième transposition, celle de la fable mythologique à la foi chrétienne. C'est ce lien qui justifie toute l'entreprise de celui qu'il convient d'appeler «translateur » plutôt que «traducteur », et qui garde pour sa dernière lecture de la fable une irréductible originalité.

Marylène Possamaï-Perez - Université Lumière-Lyon 2, UMR 5648 (CIHAM)

\section{Traduire Ovide au XIVe siècle : les amours de Mars et de Vénus au livre IV des Métamorphoses et de l'Ovide moralisé}

Cette étude recherche les sources de l'auteur anonyme qui, au début du XIV ${ }^{e}$ siècle, livre la première traduction complète en langue vernaculaire des Métamorphoses d'Ovide, avant d'en donner une deuxième " traduction ", une transposition axiologique, qui lève le voile de la fable pour en révéler le sens allégorique. Pour la traduction du latin au roman, le translateur, qui connaît le latin et peut le traduire fidèlement, choisit parfois les mots en s'aidant des gloses interlinéaires du manuscrit médiéval des Métamorphoses qu'il utilise. Mais il peut s'appuyer sur d'autres textes antiques (pour la légende de Mars, Vénus et Vulcain, il s'agit de l'Ars amatoria d'Ovide) et dispose aussi de versions romanes antérieures de la fable (en l'occurrence, celle qu'on lit dans le Roman d'Eneas). II amplifie le texte des Métamorphoses grâce à ces mêmes textes, ou grâce aux mythographes qui l'ont précédé. Mais dès cette version romane de la fable, il fait preuve d'originalité et certains détails ne se lisent dans aucun autre texte. Cependant, c'est dans la deuxième transposition, celle de l'interprétation, qu'il se détache le plus de ses sources: les deux interprétations " concrètes " se distinguent des mythographes ou des récits romanesques antérieurs par leur ampleur et leur ton. Mais c'est surtout l'u allégorie " tropologique qui se révèle d'une originalité irréductible, notamment par le ton passionné caractéristique de cet ouvrage sans doute en lien avec l'homilétique de son temps.

allégorie, fable, latin, Ovide moralisé, prédication, roman, traduction

59. Il faut préciser que bien souvent, les deux commentaires des XII ${ }^{\mathrm{e}}$ et XIII ${ }^{\mathrm{e}}$ siècles les plus utilisés, ceux d'Arnoul d'Orléans et de Jean de Garlande, sont justement mêlés, de façon mal différenciée, dans les marges des manuscrits glosés des Métamorphoses : et c'est peut-être dans son outil principal, un manuscrit semblable au Vat. lat. 1479, que le translateur du XIV siècle lit ce qui est devenu un « commentaire vulgate » aux Métamorphoses : sur cette question, voir les études savantes de F. T. Coulson, en particulier « Ovid's Metamorphoses in the school tradition of France, 1180-1400 », dans J. G. ClaRK, F. T. Coulson, K. L. McKinLey éd., Ovid in the Middle Ages, Cambridge, 2011, p. 48-82. 


\section{Translating Ovid in the fourteenth century: The love of Mars and Venus in Book IV of the Metamorphoses and the Ovide moralisé}

This article examines the sources of the anonymous author who, at the beginning of the fourteenth century, produced the first complete translation in the vernacular language of Ovid's Metamorphoses, before providing a second "translation," a thematic transposition, that lifted the veil of the fable to reveal its allegorical meaning. For the translation of the fable, the translator, who knew Latin well and could translate it faithfully, sometimes chose the words by using interlinear glosses of the medieval manuscript of the Metamorphoses he had at his disposal. However, he was also able to use other earlier texts for guidance (Ovid's Ars amatoria for the story of Mars, Venus, and Vulcan) as well as the previous Romanic versions of the fable (in this particular case, the one in the Roman d'Enéas). Thanks to these texts, or thanks to the mythographers who preceded him, the translator enhanced the text of the Metamorphoses. In this French version of the fable, he displays originality and includes certain details that are not present in any other text. However, it is in the second transposition, that of the interpretation, that he really departs from his sources: both "concrete" interpretations distinguish themselves from the writings of mythographers or from previous romantic narratives in their scale and their tone. But it is the tropological "allegory" in particular that displays an unyielding originality, in particular through the zealous tone that is characteristic of this work, which can certainly be related to the preaching of the translator's time.

allegory, fable, Latin, Ovide moralisé, preaching, Romanic, translation 